\title{
In search of the perfect indicators of left ventricular mechanical dyssynchrony
}

\author{
Salvador Borges-Neto, MD, FASNC, ${ }^{a, b, c}$ and Zainab Samad, MD, MHS \\ a Department of Radiology, Duke University School of Medicine, Durham, NC \\ ${ }^{b}$ Department of Medicine, Duke University School of Medicine, Durham, NC \\ c Division of Nuclear Medicine, DUMC, Durham, NC
}

Received May 18, 2015; accepted May 18, 2015

doi: 10.1007/s12350-015-0184-6

\section{See related article, pp. 1247-1258}

Cardiac resynchronization therapy (CRT) is indicated for the treatment of patients with advanced heart failure (HF) symptoms, a left ventricular (LV) ejection fraction $(\mathrm{EF}) \leq 35 \%$, and prolonged QRS duration. ${ }^{1}$ Unfortunately, the sole use of these current patient selection parameters leads to implantation in an estimated third of patients for whom improvement is not demonstrable. $^{2}$ Alternatively, using QRS duration as a surrogate for electrical dyssynchrony may lead to the inappropriate exclusion of large segments of the HF population that may benefit from CRT. In recent years, there has been a push towards towards investigating non-invasive imaging methods to accurately define LV mechanical dyssynchrony and thus improve patient selection for CRT.

Quantitative echocardiographic measures derived from techniques such as tissue Doppler imaging (TDI) and strain analysis have been used to identify dyssynchrony, but these methods lack reproducibility and have not consistently been able to discriminate between responders and non-responders to CRT. ${ }^{3}$ The use of phase analysis of gated single-photon emission computed tomography (SPECT) myocardial perfusion imaging (MPI) for quantifying mechanical dyssynchrony has been well described in literature..$^{4-9}$ Phase analysis using SPECT MPI can allow the simultaneous assessment of

Reprint requests: Salvador Borges-Neto, MD, FASNC, Department of Radiology, Duke University School of Medicine, Durham, NC; salvador.borgesneto@duke.edu

J Nucl Cardiol 2015;22:1259-61.

$1071-3581 / \$ 34.00$

Copyright (c) 2015 American Society of Nuclear Cardiology.
LV function, mechanical dyssynchrony, and location/ extent of scar in a proven very reproducible fashion. Therefore, it has the potential for being an ideal first test in the population of patients being considered for CRT.

Different parameters from this phase analysis technique such as peak phase, bandwidth (BW), standard deviation (SD), kurtosis, and skewness describe the characteristics of the timing of onset of myocardial contraction and thus degree and severity of LV mechanical dyssynchrony. Previous investigators evaluating the utility of phase analysis in mechanical dyssynchrony focused on using the cut-off of Phase SD of $43^{\circ}$ to define significant dyssynchrony. However, it should be noted that Phase SD is a continuous variable, while it correlates well with increasing measures of dyssynchrony such as QRS duration, its use to dichotomize populations into normal vs dyssynchronous using a single-cut point is still based on single-center data that included only patients with wide QRS duration. ${ }^{10}$ To date few investigations have focused on dyssynchrony variables as continuous measures occupying the spectrum between normal and diseased myocardium. ${ }^{11,12}$

As GSPECT MPI gains traction as a viable method for assessment of dyssynchrony, it must be evaluated for its diagnostic capabilities, prognostic power, and ultimately the clinical impact of interventions based on different GSPECT MPI-derived dyssynchrony measure cut-offs. Taking a firm step in that direction, in this issue of the journal, Romero-Farina et al. prospectively evaluated 455 patients who underwent clinically indicated GSPECT MPI. This cohort comprised normal patients defined by normal conduction, LV function, and functional capacity and a group of "abnormal patients" with either myocardial or conduction disease or both. The investigators applied an algorithm to evaluate the diagnostic or discriminant capability of the dyssynchrony indices for normal and abnormal patients, for those with conduction vs myocardial disease, and for those with indications for 
CRT. Not surprisingly, the cut-off values defining " abnormal" myocardium were relatively low with Phase SD $>18^{\circ}$ and $\mathrm{BW}>51^{\circ}$, suggesting that dyssynchrony measures may serve as sensitive indicators of myocardial disease. In addition, in this sizeable study, where a large female representation made a sex-specific analysis possible, the cut-off for dyssynchrony was found to be lower for females compared with males. The cut-offs increased for discrimination between conduction and myocardial disease and cut-offs similar to that reported by Henneman et al. were noted for patients with and without criteria for CRT.

The authors should be lauded for a detailed analysis that represents a true attempt at defining cut-offs and normal distribution of phase analysis dyssynchrony parameters derived from GSPECT MPI. They applied robust statistical methods to define dichotomy points allowing differentiation between varying levels of $\mathrm{LV}$ dysfunction. This work also underscores the fact that LV mechanical dyssynchrony probably accompanies diseased myocardium across the spectrum, i.e., larger LV volumes, lower systolic function, and wider QRS duration are associated with a wider dispersion of the time of onset of myocardial contraction.
Despite the commended work from Romero-Farina et al., the critical question remains How does one use this information in clinical practice and what does this mean for the patient in terms of outcomes? It is imaginable that a "dyssynchrony index" comprises from a combination of clinical, electrocardiographic, and non-invasive imaging parameters such as the ones from GSPECT MPI could be used to more precisely guide therapy to further improve outcomes. For example, identification of poor function with significant dyssynchrony associated with myocardial infarctions especially at the site of possible LV lead placement is unlikely to improve outcomes by CRT. Therefore, the next few steps in the search towards finding the perfect cut-offs from GSPECT MPI parameters for mechanical dyssynchrony include the use of different gold standards. These should include but not limited to response to CRT as an outcome and ultimately significant clinical events such as hospitalizations for heart failure, cardiac, and all cause mortality.

Preliminary work from Hess et al. shows that there is a relationship between dyssynchrony parameters and outcomes. In their study of over a thousand patients referred for SPECT MPI for evaluation of known or suspected CAD, increase in both cardiovascular and all

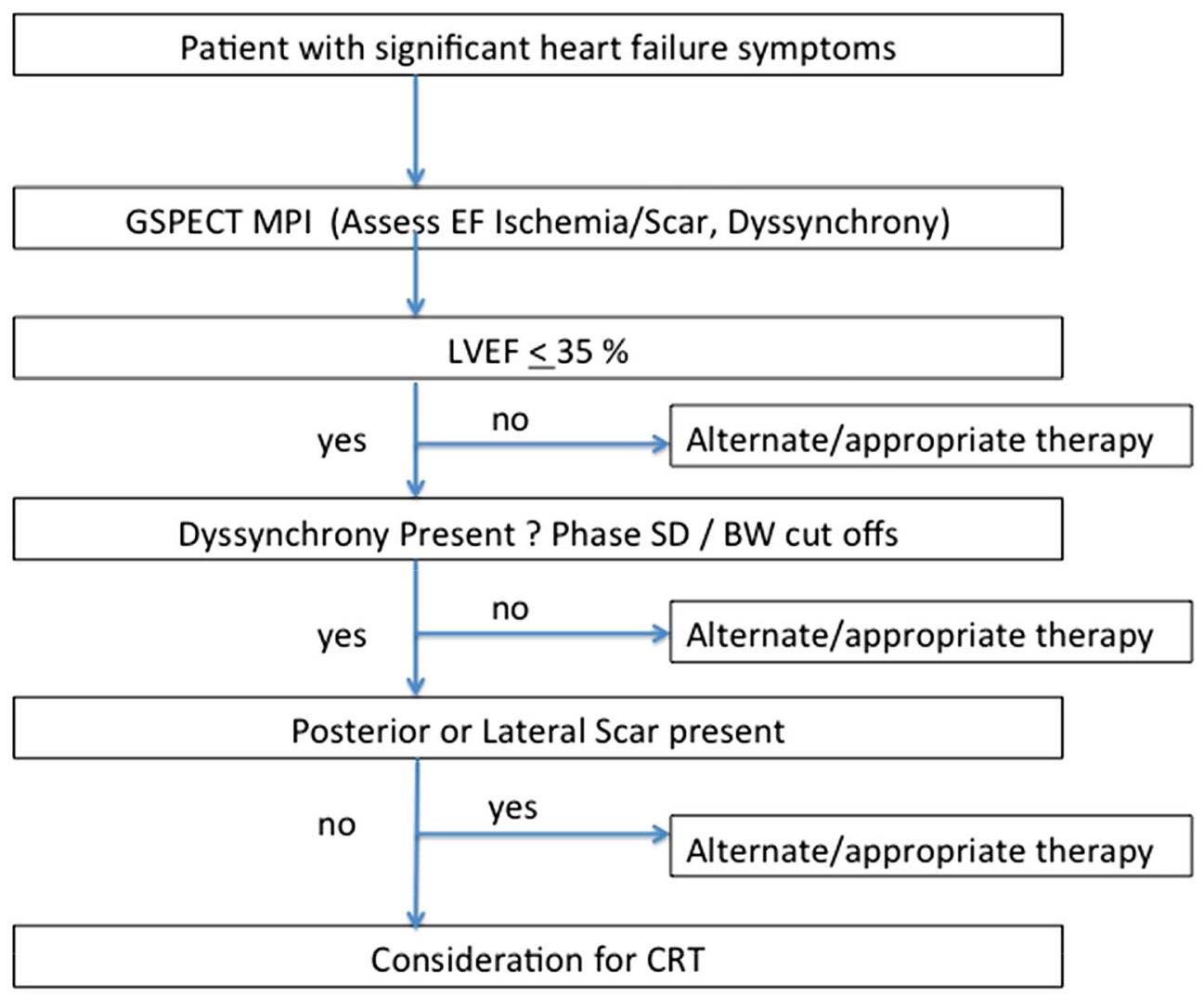

Figure 1. Proposed algorithm for use of GSPECT MPI in patient evaluation of heart failure and selection of therapies. 
cause mortality was noted with increasing in BW. Their data, similar to the work of Romero-Farina et al., support the idea of dyssynchrony representing diseased myocardium. While dyssynchrony on a continuous scale is probably useful in prognostication, for clinical decision making, cut-offs are generally helpful. The ideal model for dyssynchrony should include all parameters that SPECT MPI is capable of reporting and which carry implications for CRT implantation and response. For example, a model which includes variables such as clinical information, ejection fraction, Phase SD, BW, regions of earliest and latest contraction, presence or absence of scar, presence of scar at the site of anticipated LV lead placement must be tested for its utility in predicting response to CRT and ultimately patients outcomes (Figure 1).

\section{Disclosure}

Dr Borges-Neto received research Grant support from GE Health. Dr Samad has no relevant disclosures.

\section{References}

1. Tracy CM, Epstein AE, Darbar D, DiMarco JP, Dunbar SB, Estes NA 3rd, et al. ACCF/AHA/HRS focused update of the 2008 guidelines for device-based therapy of cardiac rhythm abnormalities: A report of the American College of Cardiology Foundation/American Heart Association Task Force on Practice Guidelines. J Am Coll Cardiol 2012;60:1297-313.

2. Abraham WT, Fisher WG, Smith AL, Delurgio DB, Leon AR, Loh $\mathrm{E}$, et al. Cardiac resynchronization in chronic heart failure. N Engl J Med 2002;346:1845-53.

3. Chung ES, Leon AR, Tavazzi L, Sun J-P, Nihoyannopoulos P, Merlino J, et al. Results of the Predictors of Response to CRT (PROSPECT) trial. Circulation 2008;117:2608-16.
4. Marsan NA, Henneman MM, Chen J, Ypenburg C, Dibbets P, Ghio S, et al. Left ventricular dyssynchrony assessed by two threedimensional imaging modalities: Phase analysis of gated myocardial perfusion SPECT and tri-plane tissue Doppler imaging. Eur J Nucl Med Mol Imaging 2008;35:166-73.

5. Boogers MM, Chen J, Bax JJ. Myocardial perfusion single photon emission computed tomography for the assessment of mechanical dyssynchrony. Curr Opin Cardiol 2008;23:431-9.

6. Chen J, Garcia EV, Henneman MM, Folks RD, Cooke CD, Faber TL, et al. Measuring left ventricular mechanical dyssynchrony from ECG-gated SPECT myocardial perfusion imaging. Minerva Cardioangiol 2008;56:227-35.

7. Chen J, Henneman MM, Trimble MA, Bax JJ, Borges-Neto S, Iskandrian $\mathrm{AE}$, et al. Assessment of left ventricular mechanical dyssynchrony by phase analysis of ECG-gated SPECT myocardial perfusion imaging. J Nucl Cardiol 2008;15:127-36.

8. Trimble MA, Borges-Neto S, Honeycutt EF, Smallheiser S, Chen $\mathrm{J}$, Shaw LK, et al. Evaluation of mechanical dyssynchrony and myocardial perfusion using phase analysis of gated SPECT imaging in patients with left ventricular dysfunction. J Nucl Cardiol 2008;15:663-70.

9. Trimble MA, Velazquez EJ, Adams GL, Honeycutt EF, Pagnanelli RA, Barnhart, et al. Repeatability and reproducibility of phase analysis of gated single-photon emission computed tomography myocardial perfusion imaging used to quantify cardiac dyssynchrony. Nucl Med Commun 2008;29:374-81.

10. Henneman MM, Chen J, Dibbets-Schneider P, Stokkel MP, Bleeker GB, Ypenburg C, et al. Can LV dyssynchrony as assessed with phase analysis on gated myocardial perfusion SPECT predict response to CRT? J Nucl Med 2007;48:1104-11.

11. Samad Z, Atchley AE, Trimble MA, Sun JL, Shaw LK, Pagnanelli $\mathrm{R}$, et al. Prevalence and predictors of mechanical dyssynchrony as defined by phase analysis in patients with left ventricular dysfunction undergoing gated SPECT myocardial perfusion imaging. J Nucl Cardiol 2011;18:24-30.

12. Atchley AE, Trimble MA, Samad Z, Pagnanelli R, Chen J, Garcia $\mathrm{EV}$, et al. Use of phase analysis of gated SPECT perfusion imaging to quantify dyssynchrony in patients with mild-to-moderate left ventricular dysfunction. J Nucl Cardiol 2009;16:888-94. 